\title{
Squeezing Operator and Squeeze Tomography
}

\author{
Octavio Castaños, Ramón López-Peña, \\ Margarita A. Man'ko* and Vladimir I. Man'ko* \\ Instituto de Ciencias Nucleares, UNAM \\ AP 70-543, 04510 México, DF, MÉXICO
}

\begin{abstract}
Some properties of Plebański squeezing operator and squeezed states created with time-dependent quadratic in position and momentum Hamiltonians are reviewed. New type of tomography of quantum states called squeeze tomography is discussed.
\end{abstract}

\section{Introduction}

Last two decades the phenomenon of squeezing, especially squeezed states in quantum optics attracted a lot of attention (see, for example [1] where the review of nonclassical states of light is presented). An important contribution to the theory of squeezed states was made by Infeld and Plebański in studies [2, 3, 4], whose results were summarized in [5]. Plebański introduced the following family of states, described by the vector:

$$
|\tilde{\psi}\rangle=\exp [i(\eta \hat{x}-\xi \hat{p})] \exp \left[\frac{i}{2} \log a(\hat{x} \hat{p}+\hat{p} \hat{x})\right]|\psi\rangle
$$

where $\hat{x}$ and $\hat{p}$ are position and momentum operators, respectively, $\xi, \eta$, and $a>0$ are real parameters, and $|\psi\rangle$ is an arbitrary initial state. Evidently, the first exponential in the right-hand side of (11) is the displacement operator written in terms of the Hermitian quadrature operators. Its properties were

\footnotetext{
${ }^{*}$ On leave from P.N. Lebedev Physical Institute, Moscow, Russia
} 
studied in 2]. The second exponential is the special case of the squeezing operator (see Eq. (2) below).

For the initial vacuum state, $\left|\psi_{0}\right\rangle=|0\rangle$, the state $\left|\tilde{\psi}_{0}\right\rangle$ (11) is exactly the squeezed state in modern terminology, whereas choosing other initial states one can obtain various generalized squeezed states. In particular, the choice $\left|\psi_{n}\right\rangle=|n\rangle$ results in the family of squeezed-number operator states, which were considered in [4, 5].

In the case $a=1$ (considered in 2]), we arrive at the states known nowadays under the name "displaced number states." Plebański gave the explicit expressions describing the time evolution of the state (11) for the harmonic oscillator with a constant frequency and proved the completeness of the set of "displaced"-number operator states.

Infeld and Plebański 3] performed a detailed study of the properties of the unitary operator $\exp (i \hat{T})$, where $\hat{T}$ is a generic inhomogeneous quadratic form of the canonical operators $\hat{x}$ and $\hat{p}$ with constant $c$-number coefficients.

Stoler [6] showed that the minimum-uncertainty states can be obtained from the oscillator ground state by means of the unitary operator depending on the complex number $z$, creation $\hat{a}^{\dagger}$ and annihilation $\hat{a}$ operators

$$
\hat{S}(z)=\exp \left[\frac{1}{2}\left(z \hat{a}^{2}-z^{*} \hat{a}^{\dagger 2}\right)\right]
$$

which was later on named the "squeezed operator." In the second paper of 6 the operator $\hat{S}(z)$ was written for real $z$ in terms of the quadrature operators as $\exp [\operatorname{ir}(\hat{x} \hat{p}+\hat{p} \hat{x})]$, which is exactly the form given by Plebański [5]. The conditions under which the minimum-uncertainty states preserve their form were studied, for example, in [7.

Plebański squeezing operator can be also used to construct the specific scheme of measuring quantum states called squeeze tomography [8]. Below we review this scheme and give a short description of other tomography methods. One of possible methods to create squeezed states is using timedependent Hamiltonians, e.g., Hamiltonian of parametric oscillator. The system with squeezing [9] and quantum damping [10] is also described by such Hamiltonians. We will illustrate the squeezing phenomenon using the example of the damped oscillator.

The quantum states are described either by wave functions [1] (pure states) or by density matrix [12, 13] (mixed states). The attempts to find a description of the quantum states which more closely resembles to the classical picture give rise to the quasidistribution functions in phase space of 
field quadratures as Wigner function [14], Sudarshan-Glauber $P$-function [15. [16], Husimi $Q$-function [17]. Recently it was understood that the states can be associated with the standard probability distribution functions. This understanding emerged when the relation between the marginal distribution function for photon homodyne quadrature (optical tomogram) and Wigner function was found [18, 19].

Optical tomography of quantum states was used to measure the quantum states of squeezed light [20, 21]. The optical tomograms depending on a rotation angle parameter were generalized [22, 23, 24] to the case of symplectic tomograms of quantum states, which depend on a field quadrature and two additional real parameters. The symplectic and optical tomograms depend on random continuous variables (homodyne quadrature components). There exists another tomographic scheme to measure the quantum states. This scheme uses probability distributions of discrete random variable $n=0,1,2, \ldots$, which has the physical meaning of number of photons [25, 26, 27]. Another tomographic scheme is based on spin tomography [28, 29, 30, 31], where discrete random variable is the spin projection $m$, $-j<m<j$.

The aim of our paper is to discuss the new tomographic representation which we called squeeze tomography. The squeeze tomogram uses the discrete random variable $n=0,1,2, \ldots$, which is the photon number analogously to the case of photon-number tomography.

The examples of the squeeze tomograms for the coherent states [16], even and odd coherent states [32, thermal states, and squeezed states [33, 34] will be considered.

\section{Squezed states of parametric oscillator and Caldirola-Kanai Hamiltonians}

Squeezed states can be generated by the parametric excitation of oscillator. In the case of parametric oscillator, the Hamiltonian is described by

$$
H=\frac{1}{2} p^{2}+\frac{1}{2} \omega^{2}(t) q^{2}
$$

where we take $\omega(0)=\hbar=m=1$. There exist the time-dependent constants of the motion which can be extracted from the Noether's theorem considering 
variations along the classical trajectories [35, 36]

$$
A=\frac{i}{\sqrt{2}}(\varepsilon(t) p-\dot{\varepsilon}(t) q),
$$

where the complex time-dependent function $\varepsilon(t)$ satisfy the classical equation of motion

$$
\ddot{\varepsilon}(t)+\omega^{2}(t) \varepsilon(t)=0,
$$

with initial conditions $\varepsilon(0)=1$ and $\dot{\varepsilon}(0)=i$, which led to satisfy the commutation relation

$$
\left[A, A^{\dagger}\right]=1 \text {. }
$$

We may find packet solutions of the Schrödinger equation which are eigenstates of operator $A$ with complex eigenvalues $\alpha$. They have the form

$$
\Psi_{\alpha}(q, t)=\Psi_{0}(q, t) \exp \left(-\frac{|\alpha|^{2}}{2}-\frac{\alpha^{2} \varepsilon^{*}(t)}{2 \varepsilon(t)}+\frac{\sqrt{2} \alpha q}{\varepsilon(t)}\right)
$$

where

$$
\Psi_{0}(q, t)=\pi^{-1 / 4} \frac{1}{\sqrt{\varepsilon(t)}} \exp \left(\frac{i \dot{\varepsilon}(t) q^{2}}{2 \varepsilon(t)}\right) .
$$

Variances of the position and momentum of parametric oscillator in these correlated coherent states can be calculated, and results are

$$
\sigma_{q}=\frac{1}{2}|\varepsilon(t)|^{2}, \quad \sigma_{p}=\frac{1}{2}|\dot{\varepsilon}(t)|^{2} .
$$

Thus, for $|\varepsilon(t)|<1$, the above states are squeezed states. The correlation coefficient $r$ of the position and momentum has the value corresponding to the minimum of the Robertson-Schrödinger uncertainty relation

$$
\sigma_{q} \sigma_{p}=\frac{1}{4\left(1-r^{2}\right)} .
$$

Then for cases where $|\varepsilon(t)|^{2}$ or $|\dot{\varepsilon}(t)|^{2}$ are less than 1 , the squeezing phenomenum is present [10].

Another example of squeezed states is related to the Caldirola-Kanai Hamiltonian, which is used to describe dissipative systems. They introduced a system described by the Lagrangian

$$
L=f(t)\left\{\frac{m}{2} \dot{q}^{2}-V(q)\right\} .
$$


In this case, the equation of motion is

$$
\ddot{q}+\frac{\dot{f}}{f} \dot{q}+\frac{1}{m} \frac{\partial V}{\partial q}=0 .
$$

The corresponding Hamiltonian is

$$
H=\frac{1}{f(t)} \frac{p^{2}}{2 m}+f(t) V(q)
$$

which can be quantized directly because it is quadratic in position and momentum operators. Generally $f(t)=\exp (2 \gamma t)$ is used to be chosen.

In the same way as we did for the parametric oscillator, we can define generalized annihilation and creation operators for the quantum case through the relation

$$
A(t)=\lambda_{q} q+\lambda_{p} p
$$

where

$$
\begin{aligned}
\lambda_{p}= & \frac{1}{\sqrt{2 \sqrt{1-\gamma^{2}}}} \exp (-\gamma t)\left\{i \exp \left(i \sqrt{1-\gamma^{2}} t\right)-\sin \left(\sqrt{1-\gamma^{2}} t\right)\right\} \\
\lambda_{q}= & \frac{1}{\sqrt{2 \sqrt{1-\gamma^{2}}}} \exp (\gamma t)\left\{\left(i \gamma+\sqrt{1-\gamma^{2}}\right) \exp \left(i \sqrt{1-\gamma^{2}} t\right)\right. \\
& \left.+\sqrt{1-\gamma^{2}} \cos \left(\sqrt{1-\gamma^{2}} t\right)-\gamma \sin \left(\sqrt{1-\gamma^{2}} t\right)\right\}
\end{aligned}
$$

The generalized coherent wave functions are given by

$$
\phi_{\alpha}(q, t)=(2 \pi)^{-1 / 4} \frac{1}{\sqrt{\lambda_{p}}} \exp \left(-\frac{|\alpha|^{2}}{2}\right) \exp \left\{-\frac{i}{2 \lambda_{p}}\left(\lambda_{q} q^{2}-2 \alpha q+i \alpha^{2} \lambda_{p}^{*}\right)\right\}
$$

The time-dependent probability distribution in position representation can be rewritten as

$$
\varrho_{\alpha}(q, t)=\frac{1}{\sqrt{2 \pi} \sigma_{q}(t)} \exp \left\{-\left(q-\frac{\left.\langle q\rangle_{\alpha}(t)\right)^{2}}{\left(2 \sigma_{q}^{2}(t)\right.}\right)\right\} .
$$

The statistical properties of these states are given by

$$
\begin{aligned}
& \langle q\rangle_{\alpha}(t)=2 \operatorname{Im}\left(\lambda_{p} \alpha^{*}\right), \quad\langle p\rangle_{\alpha}(t)=2 \operatorname{Im}\left(\lambda_{q}^{*} \alpha\right), \\
& \sigma_{q}(t)=\left|\lambda_{p}\right|^{2}, \quad \sigma_{p}(t)=\left|\lambda_{q}\right|^{2}, \quad \sigma_{p q}(t)=-\operatorname{Re}\left(\lambda_{p} \lambda_{q}^{*}\right) .
\end{aligned}
$$


The dispersion and correlation satisfy the Robertson-Schrödinger uncertainty relation $\sigma_{p} \sigma_{q}-\sigma_{p q}^{2}=1 / 4$.

Evolution of the density probability function of a coherent state under the action of the Caldirola-Kanai Hamiltonian is shown in Fig. 1.

\section{$3 \quad$ Symplectic and optical tomograms}

The state of a quantum system is described by a Hermitian trace-class nonnegative density operator $\hat{\varrho}$. For a pure state, the density operator is a projector. For continuous variables (position or field quadrature), one can introduce the optical tomographic probability distribution [18, 19, 20]

$$
\mathcal{W}_{\text {opt }}(X, \theta)=\langle\delta(X-\cos \theta \hat{q}-\sin \theta \hat{p})\rangle .
$$

This positive probability distribution, called optical tomogram, is normalised for a normalised quantum state, i.e.,

$$
\int_{-\infty}^{\infty} d X \mathcal{W}_{\mathrm{opt}}(X, \theta)=1
$$

It is important that the optical tomogram contains the same information about the states as the density operator. The optical tomogram determines completely the quantum state. It can be considered as particular characteristics of the state analogous to density matrix in position representation. The optical tomogram can be extended to become the symplectic tomogram

$$
\mathcal{W}_{\text {sym }}(X, \mu, \nu)=\langle\delta(X-\mu \hat{q}-\nu \hat{p})\rangle .
$$

Here $\mu$ and $\nu$ are real numbers. The random variable $X$ can be treated as the position (field quadrature) measured in the scaled and rotated reference frame of phase space. The symplectic tomogram also determines the quantum state completely. It can be used as characteristics of the state instead of density matrix in position (or another) representation. The parameters $\lambda$ and $\theta$, where

$$
\mu=\mathrm{e}^{\lambda} \cos \theta, \quad \nu=\mathrm{e}^{-\lambda} \sin \theta,
$$

describe scaling and rotation, respectively. Equation (24) can be rewritten using the expression for the Wigner quasidistribution function of the state $W(q, p)[14$

$$
\mathcal{W}_{\mathrm{sym}}(X, \mu, \nu)=\int \frac{d q d p}{2 \pi} W(q, p) \delta(X-\mu q-\nu p) .
$$


This expression has the inverse

$$
W(q, p)=\int \frac{d X d \mu d \nu}{2 \pi} \mathrm{e}^{i(X-\mu q-\nu p)} \mathcal{W}_{\text {sym }}(X, \mu, \nu)
$$

The density operator $\varrho$ can be expressed in terms of the symplectic tomogram $\mathcal{W}_{\text {sym }}(X, \mu, \nu)$ as follows 23 ]

$$
\hat{\varrho}=\frac{1}{2 \pi} \int d X d \mu d \nu \mathrm{e}^{i(X-\mu \hat{q}-\nu \hat{p})} \mathcal{W}_{\mathrm{sym}}(X, \mu, \nu) .
$$

The optical tomogram can be related to the Wigner function

$$
\mathcal{W}_{\text {opt }}(X, \theta)=\int \frac{d q d p}{2 \pi} W(q, p) \delta(X-\cos \theta q-\sin \theta p)
$$

\section{Squeeze tomograms}

We introduce another type of tomogram which we call squeeze tomogram $\mathcal{W}_{\text {sq }}(n, \mu, \nu)$. Here $n=0,1,2, \ldots$ has the physical meaning of the number of photons in the quantum state of light under consideration. We define the tomogram of the state with density operator $\hat{\varrho}$ by the relation

$$
\begin{aligned}
\mathcal{W}_{\mathrm{sq}}(n, \mu, \nu) & =\left\langle n\left|\hat{\mathcal{S}}(\mu, \nu) \hat{\varrho} \hat{\mathcal{S}}^{\dagger}(\mu, \nu)\right| n\right\rangle \\
& =\left\langle n\left|\hat{S}(\lambda) \hat{R}(\theta) \hat{\varrho} \hat{R}^{\dagger}(\theta) \hat{S}^{\dagger}(\lambda)\right| n\right\rangle
\end{aligned}
$$

Here $\hat{\mathcal{S}}(\mu, \nu)=\hat{S}(\lambda) \hat{R}(\theta)$, where $\hat{S}(\lambda)$ and $\hat{R}(\theta)$ are the squeezing and rotation operators, respectively. They have the form

$$
\begin{aligned}
& \hat{S}(\lambda)=\exp \left[\frac{i \lambda}{2}(\hat{q} \hat{p}+\hat{p} \hat{q})\right], \\
& \hat{R}(\theta)=\exp \left[\frac{i \theta}{2}\left(\hat{q}^{2}+\hat{p}^{2}\right)\right] .
\end{aligned}
$$

The scaling parameter $\lambda$ and rotation angle $\theta$ are connected with symplectic transform parameters $\mu$ and $\nu$ by Eq. (25).

The squeeze tomogram can be interpreted as the diagonal matrix element in a Fock basis of the scaled and rotated density operator

$$
\hat{\varrho}^{\mu \nu}=\hat{\mathcal{S}}(\mu, \nu) \hat{\varrho} \hat{\mathcal{S}}^{\dagger}(\mu, \nu) .
$$


Since the squeezing and rotation are unitary operators, the Hermitian nonnegative density operator $\hat{\varrho}^{\mu \nu}$ has positive diagonal matrix elements in the Fock basis. These matrix elements (tomograms) have the physical meaning of photon distribution functions in the state described by the density operator $\hat{\varrho}^{\mu \nu}$. To measure the tomogram one has to take the initial photon state with density operator $\hat{\varrho}$. Then one needs to rotate the quadratures as it is done in the homodyne detection scheme. The rotated state has to be squeezed by applying the squeezing operator $\hat{S}^{\dagger}(\lambda)$. Measuring the photon statistics in the obtained state with density operator $\hat{\varrho}^{\mu \nu}$ one gets the squeeze tomogram $\mathcal{W}_{\text {sq }}(n, \mu, \nu)$. This tomogram is the normalized probability distribution of the discrete random variable $n$. The tomogram is normalized, satisfying the equality

$$
\sum_{n=0}^{\infty} \mathcal{W}_{\mathrm{sq}}(n, \mu, \nu)=1
$$

The tomogram depends on the number of photons $n$ and two real parameters $\mu$ and $\nu$. The number of the parameters is sufficient to characterize the quantum state completely, since it is determined by the Wigner function depending on two real variables $q$ and $p$.

Let us find out the connection of the introduced squeeze tomogram with other characteristics of photon quantum states, e.g., with density operator (density matrix) in position representation. This connection can be presented in the form of integral transform of the density matrix

$$
\begin{aligned}
& \mathcal{W}_{\mathrm{sq}}(n, \mu, \nu) \equiv \mathcal{W}_{\mathrm{sq}}(n, \lambda, \theta) \\
& \quad=\int d x d y \varrho(x, y) \mathcal{K}(x, y, n, \mu, \nu) .
\end{aligned}
$$

The kernel of the integral transform has the form

$$
\begin{aligned}
& \mathcal{K}(x, y, n, \mu, \nu)=\frac{1}{\sqrt{\pi\left(\mu^{2}+\nu^{2}\right)} 2^{n} n !} \\
& \times H_{n}\left(\frac{x}{\sqrt{\mu^{2}+\nu^{2}}}\right) H_{n}\left(\frac{y}{\sqrt{\mu^{2}+\nu^{2}}}\right) \\
& \times \exp \left\{-i \frac{x^{2}}{2}\left[\frac{\sqrt{2}}{1-\sqrt{1-4 \mu^{2} \nu^{2}}}-\frac{\mu+i \nu}{\nu\left(\mu^{2}+\nu^{2}\right)}\right]\right. \\
& \left.+i \frac{y^{2}}{2}\left[\frac{\sqrt{2}}{1-\sqrt{1-4 \mu^{2} \nu^{2}}}-\frac{\mu-i \nu}{\nu\left(\mu^{2}+\nu^{2}\right)}\right]\right\},
\end{aligned}
$$


where $H_{n}$ denotes the Hermite polynomial of order $n$. The derivation of this formula is given in $[8$.

One can find the relation of squeeze tomogram to the Wigner function. The connection of squeeze tomogram with the Wigner function can be presented in the integral form

$$
\mathcal{W}_{\mathrm{sq}}(n, \mu, \nu)=\int d q d p W(q, p) \mathcal{K}_{W}(q, p, n, \mu, \nu)
$$

The kernel of the integral transform has the form

$$
\begin{aligned}
& \mathcal{K}_{W}(q, p, n, \mu, \nu) \\
& \quad=\frac{(-1)^{n}}{\pi} \exp \left(-|z|^{2} / 2\right) L_{n}\left(|z|^{2}\right),
\end{aligned}
$$

with

$$
\begin{aligned}
& |z|^{2}=\frac{2 q^{2}}{\mu^{2}+\nu^{2}}+2\left(\mu^{2}+\nu^{2}\right) \\
& \times\left[p-\left(\frac{\sqrt{2}}{1-\sqrt{1-4 \mu^{2} \nu^{2}}}-\frac{\mu}{\nu\left(\mu^{2}+\nu^{2}\right)}\right) q\right]^{2} .
\end{aligned}
$$

For $\nu=1, \mu=0$ ( or $\theta=\pi / 2, \lambda=0)$, which means that there is no squeezing and a $\pi / 2$ rotation, the obtained kernel coincides with the Wigner function of the Fock state (given in [35]).

The symplectic tomograms can be written within the framework of the star-product quantization [37. Then it is associated with the set of operators

$$
\begin{aligned}
& \hat{U}(\vec{x})=\delta\left(X-\hat{\mathcal{S}}^{\dagger}(\mu, \nu) \hat{q} \hat{\mathcal{S}}(\mu, \nu)\right), \\
& \hat{D}(\vec{x})=\frac{1}{2 \pi} \exp \{i(X-\mu \hat{q}-\nu \hat{p})\},
\end{aligned}
$$

with $\vec{x}=(X, \mu, \nu)$. According to the star-product quantization scheme, the symplectic tomogram is the tomographic symbol of the density operator and it is given by

$$
\mathcal{W}(\vec{x})=f_{\hat{\varrho}}(\vec{x})=\operatorname{Tr}\{\hat{\varrho} \hat{U}(\vec{x})\} .
$$

The density operator is expressed in terms of symplectic tomogram

$$
\hat{\varrho}=\int d X d \mu d \nu \mathcal{W}_{\hat{\varrho}}(X, \mu, \nu) \hat{D}(X, \mu, \nu) .
$$


Suppose that one uses the other star-product scheme described by the vector $\vec{y}=\left(n, \mu^{\prime}, \nu^{\prime}\right)$ and sets of operators $\hat{U}^{\prime}(\vec{y})$ and $\hat{D}^{\prime}(\vec{y})$. Thus we introduce the squeeze tomogram as another tomographic symbol of the density operator

$$
\mathcal{W}(\vec{y})=\phi_{\hat{\varrho}}(\vec{y})=\operatorname{Tr}\left\{\hat{\varrho} \hat{U}^{\prime}(\vec{y})\right\} .
$$

The inverse relation reads

$$
\hat{\varrho}=\sum_{n} \int d \mu^{\prime} d \nu^{\prime} \mathcal{W}_{\hat{\varrho}}\left(n, \mu^{\prime}, \nu^{\prime}\right) \hat{D}^{\prime}\left(n, \mu^{\prime}, \nu^{\prime}\right)
$$

The operator $\hat{U}^{\prime}\left(n, \mu^{\prime}, \nu^{\prime}\right)$ is given by the expression

$$
\hat{U}^{\prime}\left(n, \mu^{\prime}, \nu^{\prime}\right)=\delta\left(n-\hat{\mathcal{S}}^{\dagger}\left(\mu^{\prime}, \nu^{\prime}\right) \hat{a}^{\dagger} \hat{a} \hat{\mathcal{S}}\left(\mu^{\prime}, \nu^{\prime}\right)\right)
$$

where $\hat{a}^{\dagger}$ and $\hat{a}$ are boson creation and annihilation operators.

Two different symbols of the same operator can be related through the expression

$$
\phi_{A}(\vec{y})=\int d \vec{x} f_{A}(\vec{x}) \operatorname{Tr}\left\{\hat{D}(\vec{x}) \hat{U}^{\prime}(\vec{y})\right\}
$$

Therefore

$$
\begin{aligned}
& \mathcal{W}_{\mathrm{sq}}\left(n, \mu^{\prime}, \nu^{\prime}\right)=\int d X d \mu d \nu \mathcal{W}_{\mathrm{sym}}(X, \mu, \nu) \\
& \quad \times \mathcal{K}_{S}\left(n, \mu^{\prime}, \nu^{\prime}, X, \mu, \nu\right) .
\end{aligned}
$$

The kernel has the form 8

$$
\mathcal{K}_{\mathcal{S}}\left(n, \mu^{\prime}, \nu^{\prime}, X, \mu, \nu\right)=\frac{\mathrm{e}^{i X}}{2 \pi} \mathrm{e}^{-|\alpha|^{2} / 2} L_{n}\left(|\alpha|^{2}\right) .
$$

Here $L_{n}$ is a Laguerre polynomial and the complex variable $\alpha$ reads

$$
\alpha=\frac{1}{\sqrt{2}}(\tilde{\nu}-i \tilde{\mu}),
$$

where $\tilde{\mu}$ and $\tilde{\nu}$ are given by

$$
\begin{aligned}
\tilde{\mu} & =-\frac{\nu^{\prime}}{2 \nu}\left(1-\sqrt{1-4 \mu^{2} \nu^{2}}\right)+\mu^{\prime} \mu, \\
\tilde{\nu} & =\frac{\nu^{\prime}}{2 \mu}\left(1+\sqrt{1-4 \mu^{2} \nu^{2}}\right)+\mu^{\prime} \nu .
\end{aligned}
$$




\section{Examples}

In this section we consider several examples of squeeze tomograms of important states of photons. The first example is the ground or vacuum state of the electromagnetic field with density operator

$$
\hat{\varrho}_{v}=|0\rangle\langle 0| \text {. }
$$

The squeeze tomogram of this state reads

$$
\mathcal{W}_{0}(n, \lambda, \theta)=|\langle n|\hat{S}(\lambda)| 0\rangle|^{2},
$$

where $\hat{S}(\lambda)$ is the unitary squeezing operator. The tomogram in explicit form reads

$$
\mathcal{W}_{0}(n, \lambda, \theta)=\frac{(-\tanh \lambda)^{n}}{n ! 2^{n} \cosh \lambda}\left\{H_{n}(0)\right\}^{2} .
$$

One can see that the angle $\theta$ is not present in the tomogram for the vacuum state.

Another important state is the coherent state $|\alpha\rangle$ of the photon with the density operator

$$
\hat{\varrho}_{\alpha}=|\alpha\rangle\langle\alpha| \text {. }
$$

According to definition, the squeeze tomogram for this state reads

$$
\mathcal{W}_{\alpha}(n, \lambda, \theta)=|\langle n|\hat{S}(\lambda) \hat{R}(\theta)| \alpha\rangle|^{2}
$$

One can easily show that

$$
\langle n|\hat{S}(\lambda) \hat{R}(\theta)| \alpha\rangle=\mathrm{e}^{(\lambda+i \theta) / 2} \int d x \psi_{n}^{*}(x) \psi_{\tilde{\alpha}}\left(\mathrm{e}^{\lambda} x\right),
$$

with $\tilde{\alpha}=\alpha \mathrm{e}^{i \theta}$. We can get

$$
\begin{aligned}
& \sum_{n=0}^{\infty} \frac{\beta^{* n}}{\sqrt{n !}}\langle n|\hat{S}(\lambda) \hat{R}(\theta)| \alpha\rangle \\
& =\frac{\mathrm{e}^{i \theta / 2}}{\sqrt{\cosh \lambda}} \exp \left\{-\frac{|\alpha|^{2}}{2}+\frac{1}{2} \tilde{\alpha}^{2} \tanh \lambda\right\} \\
& \quad \times \exp \left\{-\frac{1}{2} \beta^{* 2} \tanh \lambda+\frac{\tilde{\alpha}}{\cosh \lambda} \beta^{*}\right\} .
\end{aligned}
$$


By means of the generating function of the Hermite polynomials we obtain the matrix element

$$
\begin{aligned}
& \langle n|\hat{S}(\lambda) \hat{R}(\theta)| \alpha\rangle=\mathrm{e}^{\left(-|\alpha|^{2}+i \theta+\left(\alpha \mathrm{e}^{i \theta}\right)^{2} \tanh \lambda\right) / 2} \\
& \quad \times \sqrt{\frac{|\tanh \lambda|^{n}}{2^{n} n ! \cosh \lambda}} H_{n}\left(\frac{\alpha \mathrm{e}^{i \theta}}{\sqrt{|\sinh 2 \lambda|}}\right) .
\end{aligned}
$$

For $\alpha=0$, if we take the absolute value of the last expression, we get Eq. (501). One can see that the tomograms (50) and (52) coincide with the photon distribution function of squeezed vacuum and generic squeezed coherent states, respectively. These photon distributions are given, e.g., in [35]. In Fig. 2 we illustrate the behaviour of the tomogram of a coherent state as a function of $n$ and $\lambda$, using $\alpha=3$ and $\theta=0$.

Another specific example is the Fock state of the photon $|m\rangle$ with density operator

$$
\hat{\varrho}_{m}=|m\rangle\langle m|, \quad m=0,1, \ldots .
$$

The squeeze tomogram of this state is

$$
\mathcal{W}_{m}(n, \lambda)=|\langle n|\hat{S}(\lambda)| m\rangle|^{2}
$$

In fact, it is modulus squared of the matrix element of the squeezing operator in Fock basis. It does not depend on rotation angle $\theta$. Again, the squeeze tomogram coincides with the photon distribution function of the squeezed Fock state. The example of the tomogram for the Fock state $|1\rangle$ reads

$$
\mathcal{W}_{1}(n, \lambda)=\frac{n^{2}}{2^{n-1} n !} \frac{(\tanh \lambda)^{n-1}}{(\cosh \lambda)^{3}}\left[H_{n-1}(0)\right]^{2}
$$

The even and odd coherent states (Schrödinger cat states) 32 are paradigmatic examples of superposition of quantum states. The density operators for these states read

$$
\hat{\varrho}_{\alpha}^{ \pm}=\left|\mathcal{N}_{ \pm}\right|^{2}(|\alpha\rangle \pm|-\alpha\rangle)(\langle\alpha| \pm\langle-\alpha|)
$$

where

$$
\mathcal{N}_{ \pm}=\sqrt{\frac{1}{2\left(1 \pm \mathrm{e}^{-2|\alpha|^{2}}\right)}}
$$


The squeeze tomograms for the Schrödinger cat states are

$$
\begin{aligned}
& \mathcal{W}_{\alpha}^{ \pm}(n, \lambda, \theta)=\frac{1}{1 \pm \mathrm{e}^{-2|\alpha|^{2}}}\left\{|\langle n|\hat{S}(\lambda) \hat{R}(\theta)| \alpha\rangle|^{2}\right. \\
& \left.\quad \pm \operatorname{Re}\left[\langle n|\hat{S}(\lambda) \hat{R}(\theta)| \alpha\rangle\langle n|\hat{S}(\lambda) \hat{R}(\theta)|-\alpha\rangle^{*}\right]\right\} \\
& =\frac{1}{1 \pm \mathrm{e}^{-2|\alpha|^{2}}}\left[1 \pm(-1)^{n}\right] \mathcal{W}_{\alpha}(n, \lambda, \theta) .
\end{aligned}
$$

We observe terms which are due to interference of states $|\alpha\rangle$ and $|-\alpha\rangle$.

The example of a mixed state tomogram (thermal state of light) with density operator

$$
\begin{aligned}
& \hat{\varrho}_{T}=\frac{1}{Z} \mathrm{e}^{-\frac{1}{T}\left(\hat{a}^{\dagger} \hat{a}+1 / 2\right)}, \\
& Z=\sum_{n=0}^{\infty} \mathrm{e}^{-\frac{1}{T}(n+1 / 2)}=\frac{1}{2} \operatorname{cosech}\left(\frac{1}{2 T}\right),
\end{aligned}
$$

is given by the sum

$$
\begin{aligned}
& \mathcal{W}_{T}(n, \lambda, \theta)=\frac{1}{Z} \sum_{m=0}^{\infty} \mathrm{e}^{-\frac{1}{T}(m+1 / 2)} \mathcal{W}_{m}(n, \lambda) \\
& =\frac{1}{Z} \sum_{m=0}^{\infty} \mathrm{e}^{-\frac{1}{T}(m+1 / 2)} \frac{\operatorname{sech} \lambda}{m ! n !}\left[H_{n m}^{\{\mathcal{R}\}}(0)\right]^{2}
\end{aligned}
$$

The matrix $\mathcal{R}$ is given by

$$
\mathcal{R}=\left(\begin{array}{cc}
\tanh \lambda & -\operatorname{sech} \lambda \\
-\operatorname{sech} \lambda & -\tanh \lambda
\end{array}\right) .
$$

One can see that the squeeze tomogram does not depend on the rotation angle $\theta$ because it contains the sum of Fock state tomograms, and these tomograms do not depend on the rotation angle. For $T \rightarrow 0$, the tomogram is going to the tomogram of the vacuum state.

\section{Conclusions}

We discussed different aspects of squeezing operator considered by Plebańsky long ago [2, 3, 4, 5] and its application to the problem of nonclassical (squeezed) 
states and to the problem of squeeze tomography. We have shown that for systems with time-dependent Hamiltonians, e.g., for oscillator with timedependent frequency and for damped ocsillator, the squeezed states appear naturally in the process of time evolution. We constructed also the squeeze tomogram which is a fair probability distribution of discrete random variable. The squeeze tomogram depends on extra two parameters and it describes the quantum state. The squeeze tomogram is related to other functions depending the quantum state including the Wigner function, symplectic and optical tomograms by means of integral transform. The squeeze tomography can be discussed as complimentary method to measure quantum states of photon.

\section{Acknowledgments}

M.A.M. and V.I.M. thank Institute of Nuclear Sciences of UNAM and Organizers of the Conference "Topics in Mathematical Physics, General Relativity and Cosmology on the Ocassion of the 75th Birthday of Professor Jerzy F. Plebański" for kind hospitality. M.A.M. thanks the Russian Foundation for Basic Research for partial support under Project no. 03-02-16408.

\section{References}

[1] V. V. Dodonov and V. I. Man'ko (eds.), Theory of Nonclassical States of Light, Taylor \& Francis, London \& New York (2003).

[2] J. Plebański, "Classical properties of oscillator wave packets" Bulletin de l' Académie Polonaise des Sciences, 2, 213 (1954).

[3] L. Infeld and J. Plebański, "On a certain class of unitary transformations" Acta Phys. Polon., 14, 41 (1955).

[4] J. Plebański, "On certain wave packets" Acta Phys. Polon., 14, 275 (1955).

[5] J. Plebański, "Wave functions of a harmonic oscillator" Phys. Rev., 101, 1825 (1956).

[6] D. Stoler, Phys. Rev. D, 1, 3217 (1970); 4, 1925 (1971).

[7] D. A. Trifonov, Phys. Lett. A, 48, 165 (1974). 
[8] O. Castaños, R. López-Peña, M. A. Man'ko and V. I. Man'ko, J. Phys. A: Math. Gen., 37, 8529 (2004).

[9] O. Castaños, R. López Peña, and V. I. Man'ko, Phys. Rev. A, 50, 5209 (1994).

[10] E. Padilla-Rodal, "Generalized Coherent States of Quantum Systems" Bachelor Thesis, Faculty of Sciences, UNAM (1996).

[11] E. Schrödinger, Ann. Phys. (Leipzig), 79, 489 (1926).

[12] L. D. Landau, Z. Phys., 45, 439 (1927).

[13] J. von Neumann, Mathematische Grundlagen der Quantenmechanik, Springer, Berlin (1932).

[14] E. P. Wigner, Phys. Rev., 40, 479 (1932).

[15] E. C. G. Sudarshan, Phys. Rev. Lett., 10, 277 (1963).

[16] R. Glauber, Phys. Rev. Lett., 10, 84 (1963).

[17] K. Husimi, Proc. Phys. Math. Soc. Jpn, 23, 264 (1940).

[18] J. Bertrand and D. Bertrand, Found. Phys., 17, 397 (1987).

[19] K. Vogel and H. Risken, Phys. Rev. A, 40, 2847 (1989).

[20] D. T. Smithey, M. Beck, M. G. Raymer and A. Faridani A, Phys. Rev. Lett., 70, 1244 (1993).

[21] S. Schiller, G. Breitenbach, S. F. Pereira, T. Mikker and J. Mlynek, Phys. Rev. Lett., 77, 2933 (1996).

[22] S. Mancini, V. I. Man'ko and P. Tombesi, Quantum Semiclass. Opt., 7, 615 (1995).

[23] G. M. D'Ariano, S. Mancini, V. I. Man'ko and P. Tombesi, Quantum Semiclass. Opt., 8, 1017 (1996).

[24] A. Wünsche, J. Mod. Opt., 47, 33 (2000).

[25] S. Wallentowitz and W. Vogel, Phys. Rev. A, 53, 4528 (1996). 
[26] K. Banaszeck and K. Wodkiewicz, Phys. Rev. Lett., 76, 4344 (1996).

[27] S. Mancini, V. I. Man'ko and P. Tombesi, Europhys. Lett., 37, 79 (1997).

[28] V. V. Dodonov and V. I. Man'ko, Phys. Lett. A, 229, 335 (1997).

[29] V. I. Man'ko and O. V. Man'ko, JETP, 85, 430 (1997).

[30] A. B. Klimov, O. V. Man'ko, V. I. Man'ko, Yu. F. Smirnov and V. N. Tolstoy, J. Phys. A: Math. Gen., 35, 6101 (2002).

[31] O. Castaños, R. López-Peña, M. A. Man'ko and V. I. Man'ko, J. Phys. A: Math. Gen., 36, 4677 (2003); J. Opt. B: Quantum Semiclass. Opt., 5, 227 (2003).

[32] V. V. Dodonov, I. A. Malkin and V. I. Man'ko, Physica, 72, 597 (1974).

[33] A. Vourdas and R. M. Weiner, Phys. Rev. A, 36, 5866 (1987).

[34] W. P. Schleich and J. A. Wheeler, Nature, 326, 574 (1987).

[35] V. V. Dodonov and V. I. Man'ko, Invariants and evolution of nonstationary quantum systems, Proceedings of the P.N. Lebedev Physical Institute, Vol. 183, Nova Science, New York (1989).

[36] O. Castaños, R. López-Peña and V.I. Man'ko, J. Phys. A: Math. Gen., 27, 1751 (1994) .

[37] O. V. Man'ko, V. I. Man'ko and G. Marmo, J. Phys. A: Math. Gen., 35, 699 (2002). 


\section{Figure captions}

Figure 1. Evolution of the density probability function of a coherent state with amplitude $\alpha=1 / 2$ under the action of the Caldirola-Kanai Hamiltonian with $\gamma=0.1$. In the lower part of the figure, there is a contour density plot where the phenomenon of squeezing is evident when time increases.

Figure 2. Squeeze tomogram of a coherent state. We used the parameters $\alpha=3$ and $\theta=0$, which characterize a nonrotated phase space frame. 

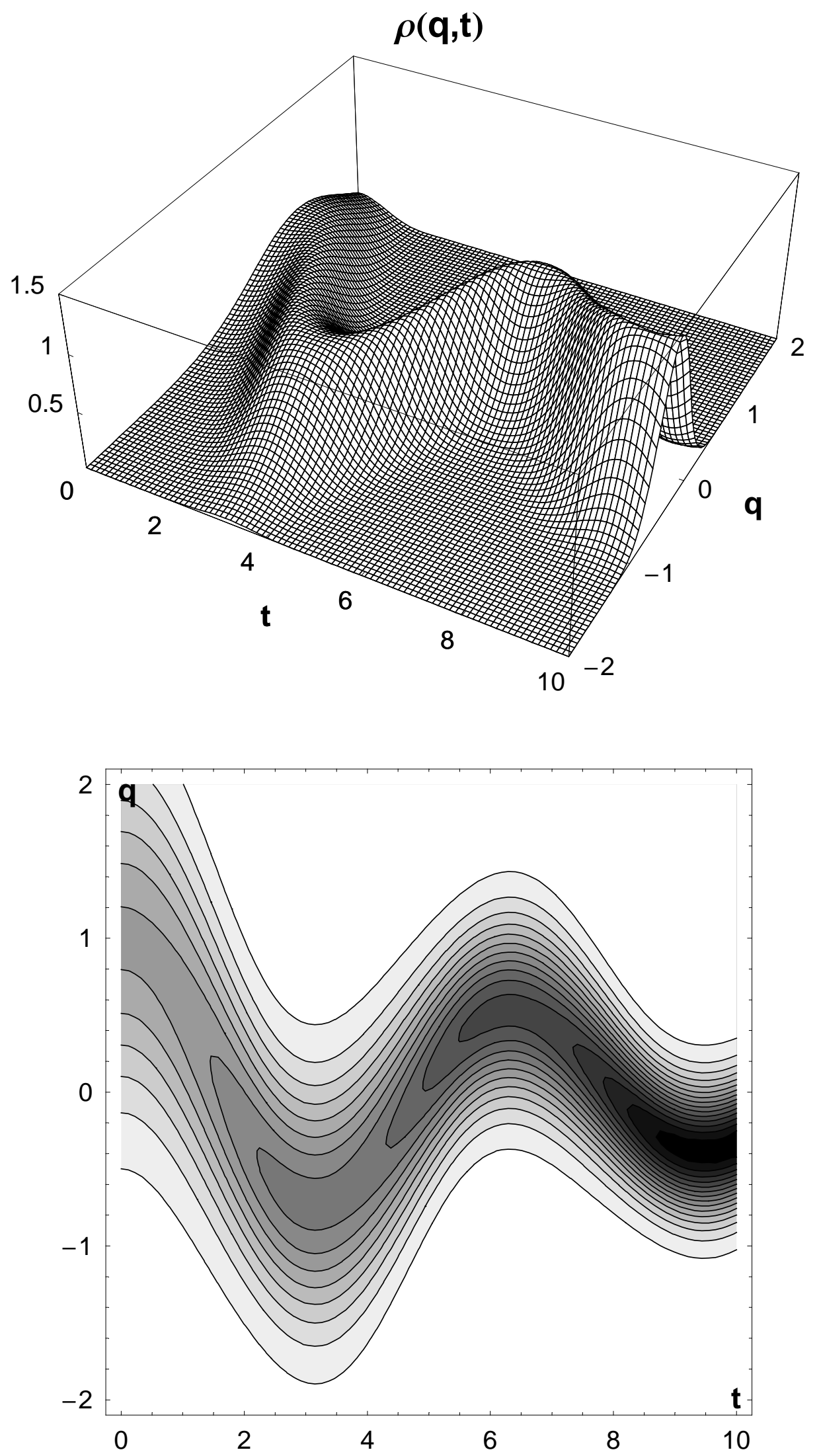


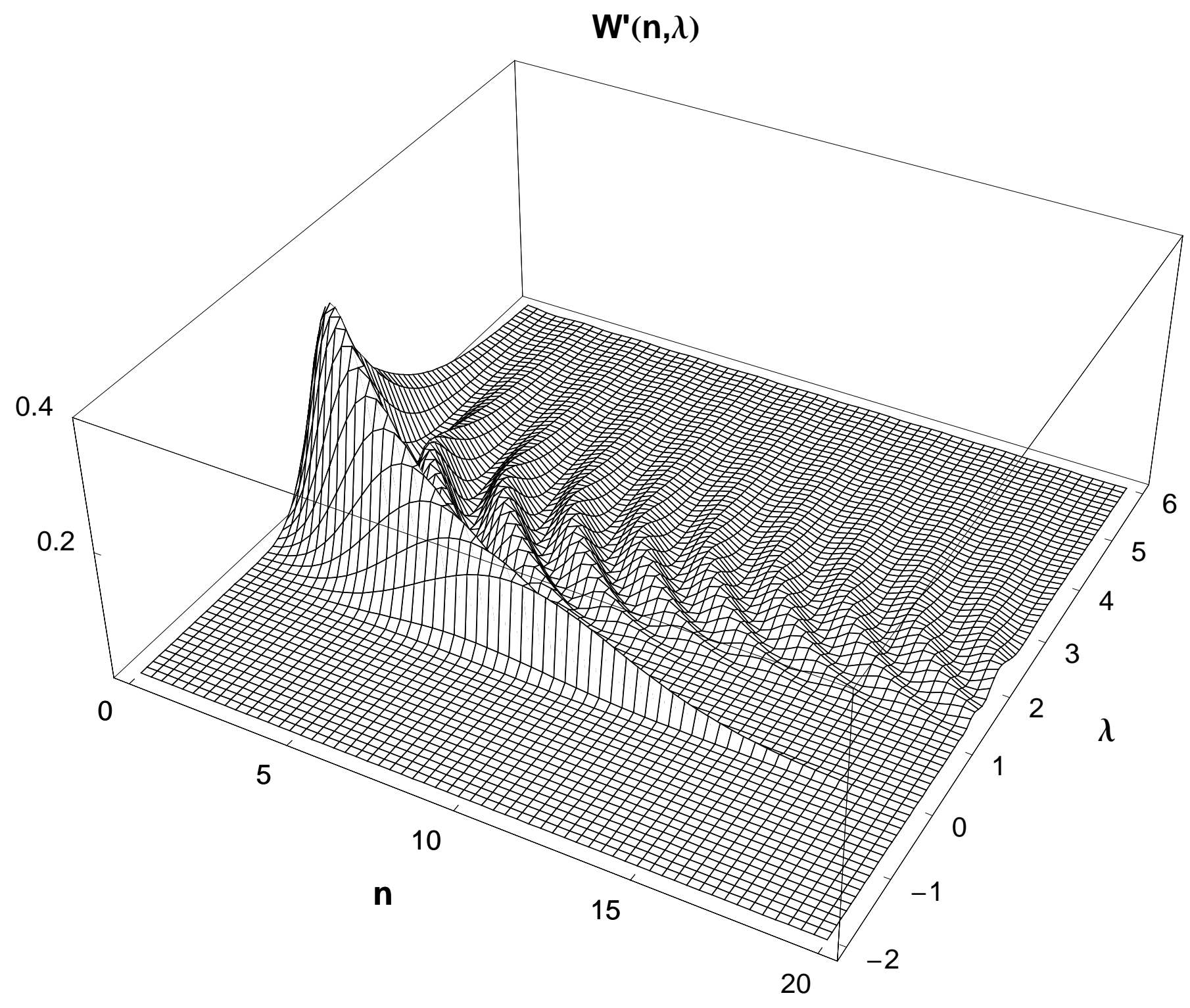

